\title{
Urinary and sexual functions after surgical treatment of penile fracture concomitant with complete urethral disruption
}

\author{
Ali Abdel Raheem, Hassan El-Tatawy, Ahmed Eissa, Abdel Hamid Elbahnasy, Mohamed Elbendary \\ Urology Department, Tanta University Hospital, Egypt.
}

\begin{abstract}
Summary Objectives: Penile fracture with concomitant complete urethral disruption is an uncommon urologic disorder. Data about the treatment and outcome measurements of this condition are scarce in the literature. The aim of the present study is to evaluate the long term urinary and sexual functions of patients with penile fracture associated with complete urethral injury after immediate surgical reconstruction.

Patients and methods: Twelve patients met our inclusion criteria and were included in this retrospective case series study; however, one was lost during follow-up. Patient's medical records were reviewed and all patients were interviewed for clinical evaluation. Urinary function was assessed by history, uroflometry and retrograde urethrography, while, sexual function was assessed by questionnaire (Sexual Health Inventory for Men) and penile Doppler for patients with erectile dysfunction.

Results: Patients' mean age was $32.3 \pm 7.5$ years (range 21-43) and the mean follow-up period was $72.6 \pm 45.4$ months (range 14-187). Vigorous sexual intercourse was the main cause in $91 \%$ of our patients. No serious long term complications was found. Only 1 patient (9\%) suffered from anterior urethral stricture, 1 patient (9\%) complained of weak erection, 3 patients (27\%) had a palpable fibrosis and 2 patients (18\%) reported a slight penile curvature during erection. Ninety one percent of all our patients maintained their normal urinary and sexual functions.

Conclusion: On the long term follow-up, most of the patients maintained their normal erectile and voiding functions with no harmful long-term complications. We advocate immediate surgical intervention and reconstruction of both corpora cavernous and urethra as a first line treatment for those patients..
\end{abstract}

KEY WORDS: Urinary function; Sexual function; Penile fracture; Surgical treatment.

Submitted 31 July 2013; Accepted 5 October 2013

\section{INTRODUCTION}

Penile fracture is a rare emergent urologic condition, which is characterized by disruption of the tunica albuginea of one corpus cavernosum or both (1). Till the year 2001, only 1331 cases had been reported in the literature (2). It seems that its incidence is higher in Middle-East countries than in USA and Western countries $(3,4)$. Penile fracture may be associated with urethral injury in up to $38 \%$ of cases (5).

Many causes of penile fracture have been reported, including sexual intercourse, sudden forced flexion, masturbation, and direct blunt trauma or rolling over in bed onto an erect penis (6). With fully erected penis, the tunica albuginea which is one of the strongest fascia in the human body - can withstand pressures up to 1500 $\mathrm{mmHg}$ - stretches and becomes as thin as $0.25-0.5 \mathrm{~mm}$ thick, while in the flaccid state it is $2.4 \mathrm{~mm}(7,8)$. So the erected penis is much more vulnerable to rupture after trauma than the flaccid penis.

Fracture penis is a clinical diagnosis, typically the patient describes hearing a popping or snapping sound followed by sudden detumescence and pain. Clinically penile swelling, hematoma, ecchymosis and penile deformity are present (Figure 1) (2).

\section{Figure 1.}

The classic clinical picture for penile fracture (swelling, hematoma and penile deformity).

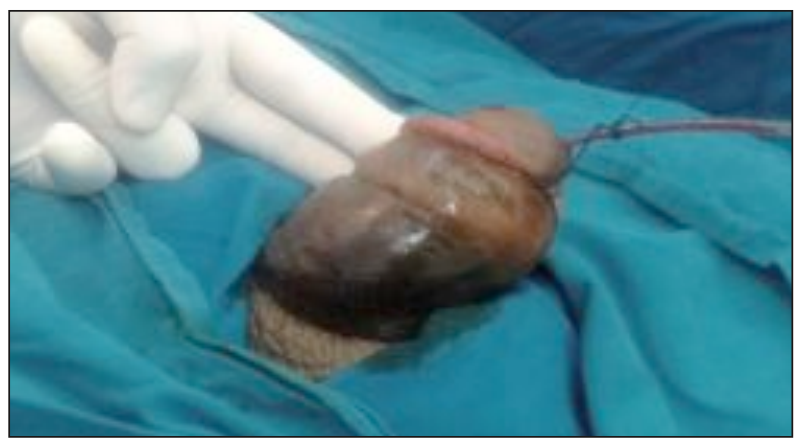


Suspicion of urethral injury is increased with presence of blood at the external meatus or hematuria (3), therefore, retrograde urethrography is prefered by many authors to confirm urethral injury diagnosis $(9,10)$.

Regarding the role of imaging studies in the diagnosis of penile fracture still there is controversy. Some studies showed the usefulness of ultrasound, cavernosography and MRI $(11,12)$ with superiority of MRI in identification of corporal injury (13). However, a recent study showed that MRI is not able to reveal detailed information about extent of corporal and urethral injury over surgical exploration (14).

For treatment of penile fracture immediate surgical exploration and repair has the advantages of short hospital stay, better patient satisfaction, and improved outcomes with lower incidence of erectile dysfunction $(6,15,16)$.

The aim of this retrospective study is to report our experience and to assess the long-term urinary and sexual functions for patients with penile fracture and complete urethral injury who were treated with immediate surgical reconstruction.

\section{Material AND MEthods}

This retrospective study was approved by our ethical committee review board. The medical records and database at our institute were reviewed to identify all patients with penile fracture who were treated surgically from 1985 till 2012. Overall 246 patients were detected of whom 34 patients had associated urethral injury (22 partial and 12 complete). We included only patients with complete urethral disruption. We reviewed the history, presentations, investigations, operative and postoperative data of these patients.

Surgical technique: Prophylactic antibiotic was used before surgery. Under spinal anesthesia all patients underwent an immediate surgical exploration through a subcoronal circumcising incision with degloving of the penis (Figure 2A). Once the site of the tunical tear was identified and the hematoma was evacuated we closed the tear with 3/0 absorbable (polydioxanone or polyglycolic acid) either continues or interrupted sutures (Figure 2B-2C). An artificial erection test through intracorporal saline injection was done to detect any leakage from the tunical tear or curvature at the repaired site. The urethra was repaired over 18 French silicon catheter. The urethral edges were dissected at both sides, trimmed, spatulated and closed with interrupted absorbable sutures using (3/0 or 4/0 polydioxanone) after ensuring tension-free end-to-end anastomosis (Figure 2D-2E). Suprapubic catheter insertion or not was determined by the surgeon preference.

Postoperative care: patients received oral antibiotics for 1 week, analgesics on demand and diazepam $5 \mathrm{mg}$ nightly in an attempt to prevent nocturnal erections. All patients were instructed to abstain from sexual activity for at least 8 weeks. The urethral catheter was left in place for at least 3 weeks and then removed. If a suprapubic catheter was inserted it was closed for at least 3 days after urethral catheter removal to ensure adequate and normal voiding before its removal.

Follow-Up: we contacted patients through the telephone
Figure 2.

Surgical technique description:

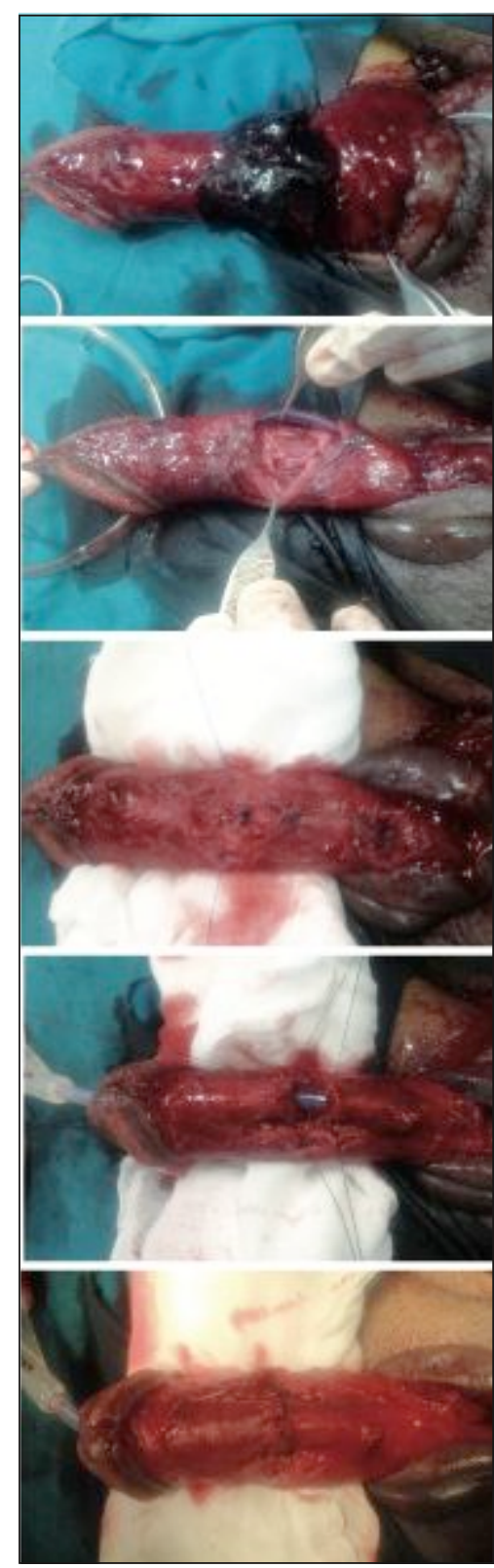

A) degloved penis with large hematoma at the fracture site.

B) After hematoma evacuation both corpora was injured with complete urethral separation.

C) After closure of the tunical tear with continuous 3/0 PDS suture.

D) The urethral edges were dissected at both sides, trimmed and spatulated with stay sutures.

E) The urethral edges were closed with interrupted absorbable sutures after ensuring tension free end-to-end anastomosis.

and an interview was done with each patient at the followup in our outpatient clinic. All patients were examined with particular concern for local penile examination to detect penile curvature and/or fibrotic nodules.

Patients' sexual function was evaluated subjectively through the Sexual Health Inventory for Men (SHIM), a questionnaire of 5 questions which is a short version of the long International Index of Erectile Function (IIEF-15) questionnaire (17).

Color Doppler ultrasonography was performed for patients with erectile dysfunction. The urinary function was evaluated objectively with uroflowmetry. Retrograde urethrography was done for all patients early after catheter removal to ensure urethral healing and during follow-up if voiding symptoms present. 
Table 1.

Patients' characteristics, perioperative and follow-up data.

\begin{tabular}{|lc|}
\hline Clinical presentation: & \\
- Penile swelling & All patients $(100 \%)$ \\
- Hematoma & All patients $(100 \%)$ \\
- Urethral bleeding & All patients $(100 \%)$ \\
- Sever pain & All patients $(100 \%)$ \\
- Acute urinary retention & 3 patients $(25 \%)$ \\
- Voiding difficulties & 4 patients $(33 \%)$ \\
\hline Predisposing factors: & \\
- Vigorous sexual intercourse & 11 patients $(91 \%)$ \\
- Forced penile pending & 1 patient $(9 \%)$ \\
\hline Perioperative data: & Mean \pm SD (Range) \\
- Age (years) & $32.3 \pm 7.5(21-43)$ \\
- Time of presentation (hours) & $5.5 \pm 3.9(1-15)$ \\
- Operative time (minutes) & $60.3 \pm 6.5(45-68)$ \\
- Hospital stay (days) & $2.1 \pm 0.7(1-3)$ \\
- Duration of catheterization (days) & $22.5 \pm 2.5(21-29)$ \\
- Follow-up period (months) & $72.6 \pm 45.4(14-178)$ \\
\hline Long term complications: & \\
- Stricture urethra & 1 patient (9\%) \\
- Erectile dysfunction & 1 patient (9\%) \\
- Palpable fibrosis & 3 patients (27\%) \\
- Penile curvature during erection & 2 patients (18\%) \\
\hline Uroflometry: & Mean \pm SD (Range) \\
- Qmax (ml/s) & $20.9 \pm 4.2(14-28)$ \\
- Voided volume (ml) & $29.9 \pm 18.5(0-70)$ \\
- PVR urine (ml) & $264.7 \pm 66.5(160-370)$ \\
\hline Sexual Health Inventory Questionnaire & Mean \pm SD (Range) \\
& $22.2 \pm 3(14-25)$ \\
\hline
\end{tabular}

\section{Figure 3.}

Showing the usual site of the tear at mid-shaft of the penis in and the urethra disruption level was opposite to and at the same tear level with retraction of urethral edges.

\section{Results}

Twelve patients who met our inclusion criteria were included in this retrospective series. All patients were married and their mean age was 32.3 years (range: $21-43$ ) and mean follow-up period was 72.6 months (Table 1 ). All patients (100\%) presented with penile swelling, hematoma and urethral bleeding on examination, while, 3 patients (25\%) and 4 patients (33\%) were presented with acute urinary retention (AUR) and voiding difficulties, respectively. The mean time elapsed between the occurrence of the trauma and the patients' arrival was 5.5 hours (range: 1-15) (Table 1).

The most common cause of penile fracture was vigorous sexual intercourse in 11 patients (91\%) while one patient reported a history of forced penile pending (Table 1 ).

A routine urethrography was done in all patients and showed extravasation of dye with loss of urethral patency at the anterior penile urethra. The site of the tear was at penile mid-shaft in all patients and the urethra disruption level was opposite to and at the same tear level (Figure 3). Suprapubic catheter was inserted in 5 patients.

The mean operative time was 60.3 minutes, the duration of catheterization ranged from 21 to 29 days, and the mean hospital stay was 2.1 days (Table 1 ).

One patient was lost during follow-up due to unknown causes and was excluded from our study. During patients' assessment a palpable fibrosis and slight penile curvature during erection were found in $27 \%$ and $18 \%$ of patients respectively. Ten out of 11 patients reported good voiding function with mean $\mathrm{Q}_{\max } 20.9 \pm 4.2$, no significant post voifing residual (PVR) urine and normal urethrography. Only one patient complained of voiding difficulites and his urethrography showed a ring stricture at the anterior urethra which was treated successfully by regular urethral dilatation (Figure 4).

Regarding the sexual function, all patients maintained normal sexual activity with complete recovery of their erectile function as shown in their SHIM with mean value $22.2 \pm 3$, except one patient who suffered from weak erection and was treated successfully with oral Sildenafil ${ }^{\circledR} 100 \mathrm{mg}$ on demand.

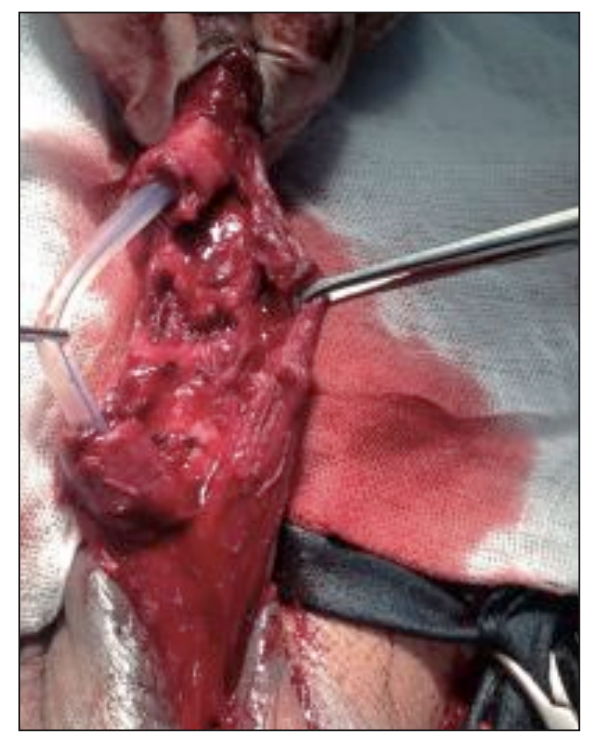

Figure 4.

A retrograde urethrogram showing a ring stricture at the anterior urethra.

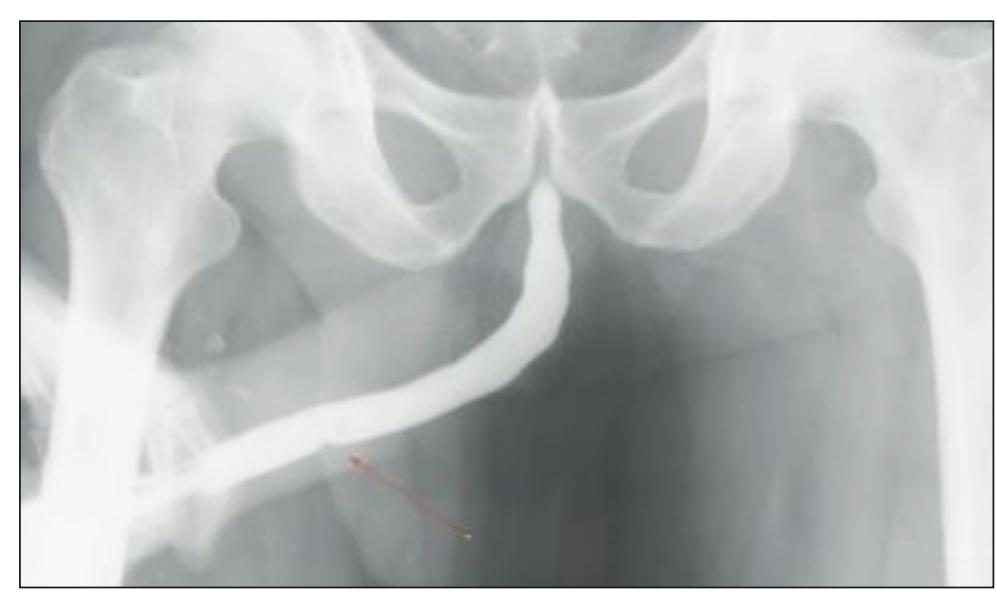




\section{Discussion}

This retrospective study is the biggest series reported in the literature for long term functional outcomes of 11 patients with penile fracture associated with complete urethral disruption. To our knowledge, only few case reports and some case series with small number of patients reported this rare condition (18-21).

In the present study the incidence of urethral injury in patients with penile fracture was 13.8\% (34/246), and this finding was in accordance with Koifman et al. who showed an incidence $12.5 \%$ for associated urethral injury (22).

The result of our study refers to both hematuria and blood at the external meatus as a hallmark findings for concomitant urethral injury diagnosis, since all of our patients (100\%) showed both findings on examination, The same findings were reported by Derouiche et al. who observed that 10 out of 10 patients (100\%) who presented with bloody urethral discharge had associated urethral injury (3). Other clinical presentations such as penile swelling, hematoma, pain and penile deformity are common findings in all patients with penile fracture, but not specific for diagnosis of urethral injury. Although $58 \%$ of our patients had voiding difficulties and AUR, however, these findings may present in the absence of urethral injury, due to the presence of penile deformity, large hematoma and severe edema causing urethral obstruction (23).

It seems to us that the association of complete urethral injury and penile fracture need more forcible trauma to occur and this was evident in our study, since, the main underlying etiology in $11 / 12$ patients was due to vigorous sexual intercourse. El-Assmy et al. reported that vigorous coital trauma was the commonest cause (50\%) of penile fracture associated with urethral injury (19). And this may also explain that all our patients had a bilateral corporal rupture owing to the severity of the trauma.

In our series all patients underwent retrograde urethrography as it is a routine investigation in our department for patients with penile fracture if a high clinical suspicion of associated urethral injury is present. The sensitivity of retrograde urethrography in the diagnosis of associated urethral injury was 100\%. In contrast, Mydlo, found that the sensitivity of this test is only $50 \%$ with a possibility of a false negative results (1).

Conservative treatment for penile fracture treatment consisted mainly of cold compresses, pressure dressings, antibiotics and anti-inflammatory drugs (24-26).

Nowadays, many studies supported the superiority of surgical treatment over conservative treatment $(27,28)$. Moreover, excellent long-term results and lower complication rates have been reported with immediate surgical repair $(1,6,29)$. The mean follow-up for our patients was 72.6 months; of them $91 \%$ showed no voiding difficulties. Several studies and case reports aroused the important role of immediate surgical repair on restoring back the normal urinary function even with complete urethral disruption $(6,19,20)$ and their findings was in match with our results. Ninety one percent maintained normal erectile function and sexual activity of our patients, whereas other studies reported similar result in $83 \%$ and $93 \%$ respectively $(19,30)$. Of the long-term complications a palpable fibrosis was found in $27 \%$ of patients and slight penile curvature on erection in $18 \%$, but this did not affect their sexual activity. A palpable penile fibrosis is a common long-term complication with an incidence ranging from $41 \%$ up to $93 \%(30,4)$.

A limitation of the present study is being a retrospective case series: in addition to, the surgical technique was not uniform regarding suprapubic catheter insertion and suturing of the tunica albuginea. However, being a retrospective study with a small number of patients may be explained by the rarity of this condition and the difficulty to be evaluated prospectively in regard to optimal initial treatment. Also it seems that the difference in some surgical steps did not affect the outcome of surgery during follow-up.

In summary, penile fracture concomitant with complete urethral rupture, although being uncommon, however, is still a urological emergency which if not managed correctly may carry the risk of many long-term complications such as erectile dysfunction, penile curvature, fibrosis and urethral stricture. To our knowledge penile fracture is diagnosed clinically, in addition, the presence of urethral bloody discharge represents an alarm for an associated urethral injury and a retrograde urethrography is recommended. The risk of concomitant complete urethral injury is increased with increase of the severity of the trauma, and most cases are due to vigorous sexual intercourse. Complete urethral disruption often present at the same level of the tunical tear and usually the tear involve both corpora. After final diagnosis we recommend immediate surgical repair of both ruptured corpora and urethra as it carries a lower risk of complication and better long-term functional outcomes for both urinary and sexual functions.

\section{Conclusion}

Penile fracture associated with complete urethral injury is a very rare, yet, an emergent urological condition.

Vigorous sexual intercourse was found to be the most common cause of concomitant complete urethral disruption. Blood at the external urethral meatus and hematuria represents a hallmark for urethral injury. Immediate surgical treatment reduces the serious long-term complications and improves the functional outcomes.

\section{RefEREnCES}

1. Mydlo JH. Surgeon experience with penile fracture. J Urol. 2001; 166:526-8.

\section{Eke N. Fracture of the penis. Br J Surg. 2002; 89:555-65.}

3. Derouiche A, Belhaj K, Hentati H, et al. Management of penile fractures complicated by urethral rupture. Int J Impot Res. 2008; 20:111-4.

4. Zargooshi J. Sexual function and tunica albuginea wound healing following penile fracture: An 18-year follow-up study of 353 patients from Kermanshah, Iran. J Sex Med. 2009; 6:1141-50.

5. Fergany AF, Angermeier KW, Montague DK. Review of Cleveland Clinic experience with penile fracture. Urology. 1999; 54:352-5.

6.Ibrahiem EI, El-Tholoth HS, Mohsen T, et al. Penile fracture: long- 
term outcome of immediate surgical intervention. Urology. 2010; 75:108-11.

7. Bitsch M, Kromann-Andersen B, Schou J, Sjontoft E. The elasticity and the tensile strength of tunica albuginea of the corpora cavernosa. J Urol. 1990; 143:642-645.

8. De Rose AF, Giglio M, Carmignani G. Traumatic rupture of the corpora cavernosa: new physiopathologic acquisitions. Urology. 2001; 57:319-22.

9. Cross M, Arnold T, Peters P. Fracture of the penis with associated laceration of the urethra. J Urol. 1977; 117: 725.

10. Zargooshi J. Penile fracture in Kermanshah, Iran: report of 172 cases. J Urol. 2000; 164:364-6.

11. Mydlo JH, Hayyeri M, Macc RJ. Urethrography and cavernosography imaging in a small series of penile fractures: a comparison with surgical findings. Urology 1998; 51:616-9.

12. Rahmouni A, Hoznek A, Duron A, et al. Magnetic resonance in penile rupture: aid to diagnosis. J Urol 1995; 153:1927-8.

13. Fedel M, Venz S, Anderssen R, et al. The value of magnetic resonance imaging in the diagnosis of suspected penile fracture with atypical clinical findings. J Urol. 1996; 155:1924-1927.

14. Hatzichristodoulou G, Gschwend J, Herkommer K, Niko Z. Accuracy of magnetic resonance imaging for diagnosis of penile fracture-comparative analysis with intraoperative finding. J Urol. 2013, 189, 4 S.

15. Nicolaisen GS, Melamud A, Williams RD, McAninch JW. Rupture of the corpus cavernosum: surgical management. J Urol. 1983; 130:917-9.

16. Asgari MA, Hosseini SY, Safarinejad MR, et al. Penile fractures: evaluation, therapeutic approaches and long-term results. J Urol. 1996; 155:148-9.

17. Rosen RC, Cappelleri JC. The Sexual Health Inventory for Men (SHIM): A 5-year review of research and clinical experience. Int J Impot Res 2005; 17:307-19.

18. El-Assmy A, El-Tholoth HS, Mohsen T, Ibrahiem EI. Long-Term
Outcome of Surgical Treatment of Penile Fracture Complicated by Urethral Rupture. J Sex Med. 2010; 7:3784-3788.

19. Tanello M, Bettini E, Griggi S, et al. A rare case of penile fracture with complete urethral rupture during sexual intercourse. Arch Ital Urol Androl. 2005; 77:153-4.

20. Soylu A, Yilmaz U, Davarci M, Baydinc C. Bilateral disruption of corpus cavernosum with complete urethral rupture. Int J Urol. 2004; 11:811-2.

21. Tsang T, Demby AM. Penile fracture with urethral injury. J Urol. 1992; 147:466-8.

22. Koifman L, Cavalcanti AG, Manes $C H$, et al. Penile fracture experience in 56 cases. Int Braz J Urol. 2003; 29:35-9.

23. Davies DM, Mitchell I. Fracture of the penis. Br J Urol. 1978; 50:426.

24. Creecy AA, Beazlie FSJr. Fracture of the penis: traumatic rupture of corpora cavernosa. J Urol. 1957; 78:620.

25. Farah RN, Stiles R Jr, Cerny JC. Surgical treatment of deformity and coital difficulty in healed traumatic rupture of the corpus cavernosa. J Urol. 1978; 120:118-20.

26. Mydlo JH, Gershbein Aband Macchia RJ. Non-operative treatment in patients with presumed penile fracture. J Urol. 2001; 165:424-425.

27. Muentener M, Suter S, Hauri D, Sulser T. Long term experience with surgical and conservative treatment of penile fracture. J Urol. 2004; 172:576-579.

28. Yapanoglu T, Aksoy Y, Adanur S, et al. Seventeen years' experience of penile fracture: Conservative vs. surgical treatment. J Sex Med. 2009; 6:2058-63.

29. Mansi MK, Emran M, el-Mahrouky A, el-Mateet MS. Experience with penile fractures in Egypt: long-term results of immediate surgical repair. J Trauma 1993; 35:67-70.

30. Ateyah A, Mostafa T, Nasser TA, et al. Penile fracture: Surgical repair and late effects on erectile function. J Sex Med. 2008; 5:1496-502.

\section{Correspondence}

Ali Abdel Raheem, MD

Hassan El-Tatawy, MD

Ahmed Eissa, MD

Abdel Hamid Elbahnasy, MD

Mohamed Elbendary, MD (Corresponding Author)

Professor of Urology

mbendary@hotmail.com

Urology Department - Tanta University Hospital, Egypt 